\title{
A SPECTRAL SEQUENCE FOR CLASSIFYING LIFTINGS IN FIBER SPACES ${ }^{1}$
}

\author{
BY J. F. MCCLENDON
}

Communicated by Walter Feit, February 9, 1968

Consider the following diagram of pointed spaces and maps

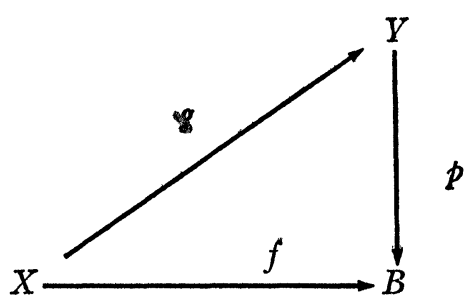

where $p g=f$ and $p$ is a fibration with fiber $F$. Suppose that $X$ is a CW-complex of dimension $\leqq 2 \operatorname{conn}(F)$ and $\operatorname{conn}(F) \geqq 1$ (conn $=$ connectivity). Let $[X, Y]_{B}$ be the set of homotopy classes of pointed maps over $f\left(H: X \times I \rightarrow Y\right.$ is a homotopy over $f$ if $p H_{t}=f$ for each $t \in I)$. Becker proved in [2], [3] that under these hypotheses $[X, Y]_{B}$ can be given an abelian group structure with $[g]$ as zero element.

The purpose of this note is to describe a spectral sequence of the Adams type which converges to $[X, Y]_{B}$. The differentials of the spectral sequence are the twisted operations described in [6], [7]. The sequence has the same relation to the method of computing $[X, Y]_{B}$ used in [6], [7] as the Adams spectral sequence has to the killing-homotopy method of computing ordinary homotopy groups. This note should be read as a sequel to [7].

A different spectral sequence for $[X, Y]_{B}$ is given by Becker in [3]. A sequence apparently similar to the one to be described here is mentioned in [4] and credited to Becker and Milgram.

1. The spectral sequence. Consider the following commutative diagram:

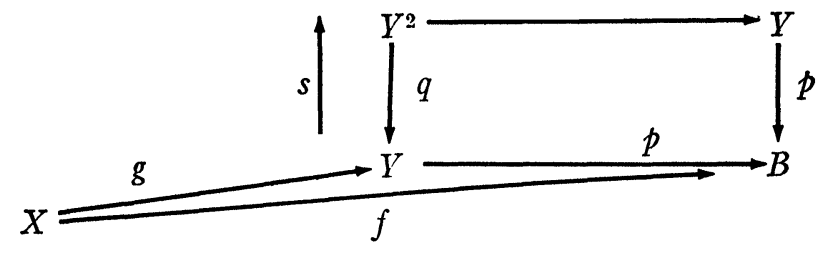

1 This research was partially supported by NSF Grant GP-6520. 
where $Y^{2}$ is the square of $Y$, i.e. the pullback of $p$ by $p$, and $s$ is the canonical cross section. Write $\left(Y^{2}, Y\right)$ for $\left(Y^{2}, s(Y)\right)$. Let $A=A_{p}$ be the $\bmod p$ Steenrod algebra and use $Z_{p}$ coefficients for all cohomology. Let $i: F \subset Y^{2}$ and assume that $i^{*}: H^{*}\left(Y^{2}\right) \rightarrow H^{*}(F)$ is onto. Assume also that $H_{j}(F ; Z)$ is finitely generated for each $j$. Let $A(Y)$ $=H^{*}(Y) \odot A$ be the Massey-Peterson algebra [5]. Then $H^{*}\left(Y^{2}, Y\right)$ and $H^{*}(X, *)$ are $A(Y)$ modules via $p: Y^{2} \rightarrow Y$ and $g: X \rightarrow Y$.

THEOREM. Under the above hypotheses, there is a spectral sequence such that

(1) $E_{2}^{s, t}=\operatorname{Ext}_{A(Y)}^{s, t}\left(H^{*}\left(Y^{2}, Y\right), H^{*}(X, *)\right)$

(2) $E_{\infty}^{s, s}=B^{s, s} / B^{s+1, s+1}$, where $[X, Y]_{B}=B^{0,0} \supset B^{1,1} \supset B^{2,2} \supset \ldots$

and $\cap B^{s, s}=$ all elements of $[X, Y]_{B}$ of finite order prime to $p$.

Notes. (1) $H^{*}\left(Y^{2}, Y\right)$ can be easily computed as an $A(Y)$ module in terms of $H^{*}(Y)$ by the results of [5].

(2) Low level computations with the spectral sequence are not difficult. However, the results can be obtained also, and sometimes more easily, by the methods of [6], [7]. The spectral sequence should ultimately prove valuable for proving general theorems about $[X, Y]_{B}$ (e.g., about immersion groups).

(3) If $B=*$ (a point) then the spectral sequence reduces, after a little manipulation of $E_{2}$, to the Adams spectral sequence for $[X, Y]$.

2. Sketch of the proof. Let $\xi Y$ be the category of all triples $(Z, Z, z)$ where $Y \stackrel{z}{\rightarrow} Z \stackrel{\vec{z}}{\rightarrow} Y$ and $z \dot{z}=1$, i.e., of all coretractions of $Y$ with given retraction. A morphism in the category is a map $m: Z \rightarrow W$ such that $m z=\check{w}$ and $w m=z$. Recall from [6], [7] that one can define a notion of homotopy in $\Im Y$ (in the obvious way) and also cone, suspension, path, and loop functors enjoying the same properties as the usual functors on $3 *$ ( $=$ the ordinary category of pointed spaces and maps). The cone-suspension sequence (Puppe sequence) and the path-loop sequence are exact after application of $\langle-, Z\rangle$ and $\langle Z,-\rangle$ respectively. $\langle-,-\rangle$ denotes the set of homotopy classes of maps in the category. In brief, all the notions concerning $\Im *$ generalize to $\Im Y$.

We will now apply an upside down version of Adams' method [1] to $q: Y^{2} \rightarrow Y$. Since $[X, Y]_{B}=\left[X, Y^{2}\right]_{Y}=\left\langle X \vee Y, Y^{2}\right\rangle$, we can work in $\Im Y$. Suspension of $Y^{2}$ in $\Im Y$ has the effect of suspending $F$ in $J *$. Successively larger pieces of the spectral sequence are obtained by taking successively higher suspensions of $Y^{2}$. We will be content here with one piece. Assume conn $(F)=n$. Consider the following commutative diagram in $3 *$. 


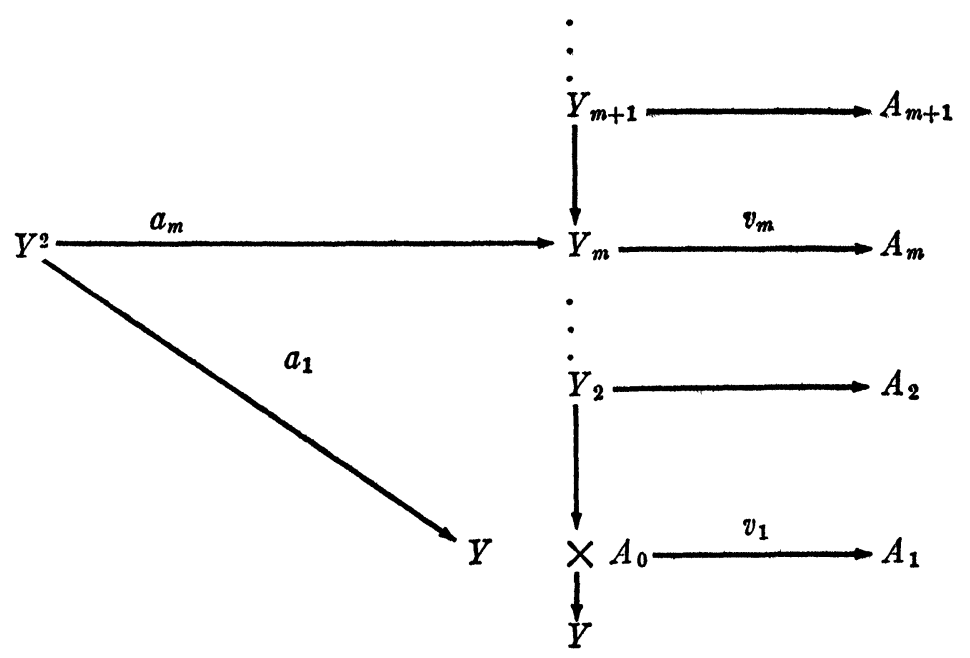

Each $A_{i}$ is a product of $K\left(Z_{p}, j\right)$ 's. $a_{1}=(q, u)$, where $u=\left(u_{1}, u_{2}, \cdots\right)$ and the $i^{*} u_{j}$ 's form a set of $A$ generators for $H^{j}(F), j \leqq 2 n+1$. $v_{m}=\left(v_{m, 1}, v_{m, 2}, \cdots\right)$ and the $v_{m, j}$ 's form a set of $A(Y)$ generators for $\left(\operatorname{ker} a_{m}^{*}\right)^{j}, j=2 n+1$.

The tower can be formally written as a new tower in $5 Y$ simply by replacing $A_{m}, m>0$, by $Y \times A_{m}$ and $v_{m}$ by $\left(q_{m}, v_{m}\right)$ where $q_{m}: Y_{m} \rightarrow Y$ is from the original tower. Each fibration $Y_{m} \rightarrow Y_{m-1}$ is a fibration in $\Im Y$ induced from a principal fibration in $3 Y$.

Now apply the functor $\langle X \vee Y,-\rangle$. The resulting exact couple gives the promised piece of the sequence.

\section{REFERENCES}

1. J. F. Adams, On the structure and applications of the Steenrod algebra, Comment. Math. Helv. 32 (1958), 180-214.

2. J. C. Becker, Homotopy theory of cross-sections and equivariant maps in the stable range, Thesis, University of Michigan, 1964.

3. - Cohomology and the classification of liftings (to appear).

4. A. Liulevicius, The cohomology of Massey-Peterson algebra (to appear).

5. W. Massey and F. Peterson, The cohomology structure of certain fiber spaces. I. Topology 4 (1964), 47-66.

6. J. F. McClendon, Higher order twisted cohomology operations, Thesis, Univ. of California, Berkeley, 1966.

7. - Higher order twisted cohomology operations (to appear).

\section{YALE UNIVERSITY}

